\title{
Criminologie
}

\section{La surveillance en libération conditionnelle, plaidoyer pour un changement d'orientation}

\section{André Précourt et Jacques Racicot}

Volume 14, numéro 2, 1981

Libération conditionnelle : évolution et application (1899-1981)

URI : https://id.erudit.org/iderudit/017137ar

DOI : https://doi.org/10.7202/017137ar

Aller au sommaire du numéro

Éditeur(s)

Les Presses de l'Université de Montréal

ISSN

0316-0041 (imprimé)

1492-1367 (numérique)

Découvrir la revue

Citer cet article

Précourt, A. \& Racicot, J. (1981). La surveillance en libération conditionnelle, plaidoyer pour un changement d'orientation. Criminologie, 14(2), 7-24.

https://doi.org/10.7202/017137ar d'utilisation que vous pouvez consulter en ligne.

https://apropos.erudit.org/fr/usagers/politique-dutilisation/ 


\title{
LA SURVEILLANCE EN LIBERATION CONDITIONNELLE, PLAIDOYER POUR UN CHANGEMENT D'ORIENTATION
}

\section{A) LA SITUATION}

\author{
André Précourt \\ Jacques Racicot
}

La surveillance des libérations conditionnelles est un processus de resocialisation qui consiste à aider le libéré conditionnel ou le détenu placé sous surveillance obligatoire dans ses efforts pour vivre au sein de la communauté comme un citoyen respectueux de la loi dans le cadre des modalités et des conditions de son régime de libération'.

Voilà la définition fondamentale du travail que devrait exercer tout agent de libération conditionnelle qui entre en fonction sous la tutelle du Service correctionnel canadien.

C'est à cet agent que sera confiée la resocialisation de tous les individus qui quittent le pénitencier. Seuls les détenus libérés moins de 60 jours avant la fin de leur sentence et ayant à leur crédit moins de 60 jours de rémission gagnée n'auront pas à se présenter à cet agent de libération conditionnelle. Toutefois, étant donné que la peine minimale est de deux ans pour être admissible à une détention dans un pénitencier, le détenu, qui quittera le lieu de détention avec moins de 60 jours à purger, devra avoir sa libération conditionnelle ou sa surveillance obligatoire révoquée et ainsi avoir côtoyé l'agent de libération conditionnelle à une ou plusieurs reprises antérieurement au cours de cette même sentence ${ }^{2}$.

\section{Les normes}

Que ce soit en libération conditionnelle, celle-ci survenant entre le tiers et les deux tiers de la sentence, ou en surveillance obligatoire (sortie après les deux tiers de la sentence), un agent sera donc rattaché à la surveillance de l'ex-détenu. Comme nous l'avons esquissé précédemment, cette relation obligatoire comporte certaines attentes de la part de l'employeur. Celui-ci mentionne trois fonctions spécifiques de l'agent :

Le surveillant des libérations conditionnelles [...] s'occupe d'assurer aux libérés conditionnels et aux détenus placés sous surveillance obligatoire :

1. Service correctionnel canadien, Manuel des procédures du S.N.L.C., p. 1.

2. L'individu, qui voit sa libération conditionnelle ou sa surveillance obligatoire révoquée, soumet la partie de sa sentence non expirée à un nouveau calcul où il devra purger entre le tiers et les deux tiers des jours non écoulés au pénitencier. 
a) l'aide pour trouver un logement, un emploi, une assistance pécuniaire, s'il y a lieu, etc.;

b) l'appui dont très peu de libérés conditionnels peuvent ou pourraient, à un moment donné, se passer lorsqu'ils se retrouvent devant des relations chancelantes sur le plan familial, des difficultés sur le plan du travail et des autres rapports sociaux, ou qu'ils se heurtent à d'autres genres de déceptions et de problèmes;

c) une surveillance adéquate de leurs activités et de leurs allées et venues.

Le surveillant se doit autant d'effectuer un contrôle que de fournir un service et d'apporter un appui. Il doit jouer le rôle d'un catalyseur de changement en offrant aide et appui aux détenus, et également en exerçant un contrôle ${ }^{3}$.

L'agent de libération conditionnelle doit donc apporter une aide à l'ex-détenu tout en exerçant une surveillance qui deviendra fréquemment cœrcitive à plusieurs égards. En effet, que ce soit de ne pas autoriser un libéré à se rendre dans une autre région sans en faire la demande, de l'obliger à se rapporter mensuellement au poste de police de son quartier ou de suspendre sa libération parce qu'il vient de commettre un délit, tout agent aura à exercer une contrainte à l'égard de chacun de ses libérés en certaines occasions. Cependant, il est évident qu'il y a des ex-détenus qui acceptent plus facilement que d'autres de se plier aux normes qu'ils doivent respecter.

D'autre part, puisqu'une certaine latitude est laissée à l'agent, plusieurs percevront leur rôle beaucoup plus en termes d'aide thérapeutique, d'autant plus que la majorité des agents du Québec ont une formation en sciences humaines. Par contre, d'autres surveillants exerceront leur fonction sous l'égide du contrôle, même si cela est moins fréquent, étant donné l'ingratitude de cette dimension dans la relation avec le libéré. Enfin, la majorité des agents se situeront entre ces deux pôles d'attraction selon leur perception du libéré, leur formation antérieure, leur objectif premier, etc. Toutefois, la couleur personnelle qu'accordera l'agent à cette fonction ne pourra l'éloigner de cette dualité entre l'aide apportée au libéré et le contrôle qu'il doit exercer.

La clientèle de l'agent se compose de libérés conditionnels qui ont volontairement accepté de quitter l'institution avant leur terme d'emprisonnement afin de purger la fin de leur sentence dans la société et dont la Commission des libérations condition-

3. Service correctionnel canadien, op. cit., p. 2. 
nelles a accepté le projet de sortie. Ces personnes sont en général conscientes de la chance qui leur est offerte et sont généralement prêtes à se conformer aux conditions auxquelles elles sont soumises afin de conserver la liberté. Ce sont également, en majorité, des détenus ayant le moins de délits à leur actif.

Depuis 1970, l'ex-détenu en surveillance obligatoire doit purger le dernier tiers de sa sentence, qu'il a gagné lors de sa période d'incarcération, dans la communauté. Il est généralement mécontent de cette situation et ne s'y conforme que parce qu'il veut demeurer en liberté. Toutefois, les normes de surveillance sont identiques dans les deux cas et s'énoncent ainsi :

1. Demeurer jusqu'à l'expiration de la sentence sous l'autorité du représentant désigné par la Commission nationale des libérations conditionnelles.

2. Se rendre directement et immédiatement à l'endroit spécifié dans les instructions et dès l'arrivée se rapporter au surveillant et ensuite à la police selon les instructions du surveillant.

3. Demeurer dans les environs immédiats, tel que désigné, et ne pas quitter ce territoire avant d'obtenir au préalable, par l'entremise du surveillant, la permission du représentant de la Commission nationale des libérations conditionnelles.

4. S'efforcer de travailler régulièrement et faire part immédiatement au surveillant de tout changement ou cessation d'emploi ou tout autre changement de circonstances comme un accident ou la maladie.

5. Obtenir au préalable l'autorisation du représentant de la Commission nationale des libérations conditionnelles par l'entremise du surveillant avant de :

a) faire l'achat d'une automobile;

b) contracter des dettes par emprunt d'argent ou par achat à tempérament;

c) assumer des responsabilités additionnelles, comme le mariage;

d) posséder ou avoir en sa possession une arme à feu ou toute autre arme.

6. Communiquer immédiatement avec le surveillant ou le représentant de la Commission nationale des libérations conditionnelles si arrêté ou interrogé par un officier de police au sujet d'un délit quelconque.

7. Obéir à la loi et s'acquitter de toutes les responsabilités légales et sociales.

4. Service correctionnel canadien, op. cit., p. 1 à 14. 


\section{Dichotomie de la fonction de l'agent}

La dichotomie du rôle joué par l'agent et les caractéristiques individuelles de chaque ex-détenu provoqueront diverses situations de tension. La plus apparente sera l'imposition de normes identiques aux libérés conditionnels et à ceux qui quittent l'institution en surveillance obligatoire. Ceux-ci ne prisant pas le fait de se soumettre à des normes semblables à celles qui régissent les libérés conditionnels, la relation avec l'agent sera généralement assez agressive dès le départ. Le surveillant se verra donc dans l'obligation de jouer la carte du pouvoir et du respect des conditions auxquelles l'ex-détenu doit être assujetti et ainsi la relation s'élaborera sur une base d'animosité même si graduellement cette relation s'améliorera ou se détériorera davantage selon le comportement des deux interlocuteurs.

Il est fréquent que l'agent soit mécontent de devoir prendre une telle attitude d'autorité qui l'oblige à vivre des situations où la méfiance domine et où il ne se sent pas à l'aise. Cependant, l'agent joue un rôle spécifique dans une structure où il doit appliquer des normes qu'il ne peut changer, une politique qui s'élabore beaucoup plus facilement en termes de contrôle que de relation d'aide et où son travail est évalué en fonction du respect de normes factuelles (nombre de rencontres, remise des différents rapports, respect de la bureaucratie, etc.). Alors qu'à son arrivée dans cette fonction, l'agent se voyait d'abord en tant qu'intervenant dans la réinsertion sociale de l'ex-détenu, il constate qu'environ deux heures sur trois sont consacrées à la rédaction de rapports qui trop souvent ne serviront jamais.

Plusieurs options s'offrent aux agents travaillant dans ce secteur. Certains respecteront les critères minimaux qui sont exigés, d'autres tenteront en toute bonne foi d'aider le mieux possible les libérés qu'on leur confie alors qu'un autre groupe voudra absolument exercer les notions de thérapie qu'ils ont acquises antérieurement. Paradoxalement, c'est sur cette capacité de relation d'aide que sera évalué Je futur agent des libérations conditionnelles lors de son embauche. N'y aurait-il donc pas un certain conflit entre cette idéologie du traitement mise de l'avant au début des années soixante et une structure administrative qui doit sans cesse prouver le nombre de ses interventions et son besoin d'effectifs?

L'agent devient donc le point de contact privilégié pour l'exdétenu. C'est lui qui présentera le libéré à ses supérieurs et propo- 
sera le cheminement à suivre ou le geste à poser en situation de crise. C'est aussi l'agent qui agira en tant qu'informateur et modérateur vis-à-vis la répression policière souvent très forte envers les ex-détenus, les créanciers qui attendent depuis trop longtemps pour être payés, les employeurs qui trouvent toujours une bonne excuse pour ne pas embaucher ces personnes, la population qui les rejette trop souvent et les normes que le libéré transgresse volontairement. Par contre, la famille du détenu excusera généralement l'inculpé et percevra à tort ou à raison l'agent comme un membre du système répressif, les organismes bénévoles se feront souvent les bienfaiteurs du libéré qui profitera le plus possible de ces derniers et les amis de l'ex-détenu lui proposeront de devenir membre d'un complot dès sa sortie du pénitencier.

- Policier

- Famille de l'ex-détenu

- Créancier

- Employeur

- Population

Agent

- Normes

Ex-détenu

C'est dans ce milieu conflictuel et en relation avec l'exdétenu que l'agent tentera d'inculquer certaines valeurs à celui-ci afin de lui permettre de ne plus réintégrer le milieu carcéral. En effet, tous les agents gravitant autour de l'ex-détenu se référeront tôt ou tard à l'agent qui devra rapidement prendre position, tempérer la situation et poser les gestes nécessaires s'il y a lieu.

L'agent tente donc d'accompagner le libéré dans toutes ces démarches. Si le premier oublie facilement le caractère obligatoire de ces rencontres, le second s'y réfère constamment, principalement quand il se bute aux mesures de contrôle.

Le contexte de rencontres obligatoires, d'objectif thérapeutique doublé d'un contrôle permanent incite l'agent de libération conditionnelle à s'interroger sur la possibilité de concilier ces objectifs au niveau d'une relation inter-personnelle.

B) LE THÉRAPEUTE ET LA THÉRAPIE

Du fraudeur de documents officiels au libéré sans famille, sans emploi, possédant une scolarité de cinquième et ayant cinquante dollars, qu'il gaspille à sa sortie du pénitencier, s'étend toute une gamme d'ex-détenus ayant des caractéristiques aussi diverses que complexes.

Très souvent, le premier aura des conditions matérielles lui permettant, s'il le veut, de quitter les milieux défavorisés et trop 
souvent délinquants. Par contre, le second ne pourra se trouver d'emploi, ou alors deviendra «bus-boy» au premier restaurant qui voudra bien l'embaucher. Entre sa petite chambre mal désinfectée du quartier pauvre de la ville et son emploi peu revalorisant, il fréquentera la taverne la plus proche de son lieu de résidence. Avec ses $160 \$$ par semaine, peut-il avoir un autre mode de vie? Ou alors, sans emploi, ses prestations du B.E.S. s'élevant à environ $330 \$$ par mois, ou $120 \$$ s'il n'a pas 30 ans, il occupera ses journées à visiter son frère dernièrement remercié de ses services à l'usine de sacs à main et qui demeure dans un logement de cinq pièces avec son épouse et ses trois enfants. À une extrémité, le gérant d'un petit commerce qui a été inculpé pour fraude, à l'autre, le libéré démuni, sans ressource, incapable de planifier un budget sur une période d'un mois. Tous, oui tous, auront droit au contrôle et à la thérapie octroyés avec bonne volonté par l'agent de libération conditionnelle.

Est-ce que le passage d'un individu au pénitencier justifierait à Iui seul l'usage de la thérapie? Bien que le milieu carcéral soit pénible à vivre, et nous en convenons très bien, il ne nous apparaît pas que la simple présence dans un pénitencier pendant une période de huit mois ou deux ans nécessite une relation thérapeutique à plus ou moins long terme.

D'autre part, si la déviance par rapport à une loi implique nécessairement un besoin thérapeutique, que ferons-nous avec tous ceux qui doivent payer des contraventions pour excès de vitesse, ceux qui n'écopent que d'une sentence suspendue ou alors tous ceux qui commettent des délits mais qui ne sont pas arrêtés puis condamnés? Nous ne croyons donc pas que le passage dans un pénitencier justifie à lui seul une thérapie prolongée.

L'attitude des libérés corrobore bien notre premier diagnostic. En effet, tout agent de libération conditionnelle, qui a quelque expérience, a été témoin des nombreuses boutades des ex-détenus. Je me souviens bien du libéré que nous allions voir chez lui et dont le frère s'exclama : "Prends le divan, ton psychiatre arrive *. Les libérés qui nous ont exprimé le besoin et non l'obligation de parler de leurs problèmes personnels autres que matériels (argent, logement, emploi, etc.) sont peu nombreux. Il suffit de relever le nombre d'ex-détenus qui viennent nous rencontrer librement et par désir personnel sans nous demander comment acquérir de l'argent ou un emploi pour nous rendre à l'évidence que l'ex- 
détenu n'exprime pas le désir de «subir » une thérapie. À l'opposé, l'agent veut trop souvent imposer, malgré la volonté du libéré de quitter le bureau de libération conditionnelle le plus rapidement possible, une relation «thérapeutique » dont le premier se moque éperdument.

Il n'est pas dans notre intention de nier tout besoin de thérapie. Il est vrai, particulièrement pour les détenus qui quittent le pénitencier après une longue période d'incarcération, que l'entrée dans la société est très difficile et que sans une aide thérapeutique adéquate, le libéré aura de la difficulté à effectuer des tâches qui paraissent très anodines au citoyen moyen, ou aura des comportements inappropriés au vécu social.

Mais cette situation est relative à une faible proportion des libérés. C'est alors que l'agent de libération conditionnelle se sentira mal équipé pour répondre à ce problème et se tournera généralement vers des soins spécialisés (clinique externe, centre d'accueil, etc.).

La thérapie que l'agent offre au détenu semble donc inappropriée aux besoins d'un petit nombre et non désirée par la majorité. Quels sont les facteurs à la base de ce problème?

\section{L'ambiguïté du rôle du clinicien}

Dès sa sortie du pénitencier, le libéré affrontera une première fois son agent de libération conditionnelle où ce dernier lui mentionnera des conditions qu'il devra suivre jusqu'à la fin de sa sentence hors-carcérale tout en proposant son aide dès que l'exincarcéré en ressentira le besoin.

Dès ce moment, le libéré aura confirmé ce que d'autres incarcérés lui ont dit à de multiples reprises alors qu'il préparait cette entrée dans la communauté. L'agent de libération conditionnelle est membre d'un système répressif, au même titre que le policier ou le garde de prisons. Son action demeure la suite logique de celle de l'agent de gestion de cas institutionnel à qui le détenu racontait ses problèmes au pénitencier, mais également à qui il cachait ses infractions par crainte d'un mauvais « rapport 》 pouvant lui valoir une punition ou, pire, le prolongement de sa période d'incarcération. Bref, le libéré sera méfiant vis-à-vis de son agent de libération conditionnelle et, quoiqu'il l'utilise à l'occasion pour se chercher un emploi ou pour répondre à des exigences de sa libération, il tentera de lui cacher une grande partie de ses activités, 
principalement celles qui s'associent facilement avec la déviance et évidemment tout acte illégal.

La majorité des libérés rencontreront donc leur agent à toutes les deux semaines comme le stipule une des conditions de leur libération. Plusieurs d'entre eux viendront uniquement faire poinçonner leur carte et chercheront à quitter les lieux le plus rapidement possible alors que d'autres, qu'on qualifiera souvent de moins criminalisés, accepteront un dialogue courtois avec leur agent, allant même jusqu'à entreprendre certains sujets de discussion. Toutefois, ils se garderont bien de mentionner qu'ils auraient peut-être effleuré de près la déviance par rapport aux normes sociales. Devant l'agent de libération conditionnelle, le libéré est toujours un "buveur social », il ne consomme de la drogue que très rarement, il n'a jamais de bataille avec un autre habitué de la taverne du coin et, évidemment, il n'a jamais transgressé la loi sans se faire arrêté ou sans se faire prendre sur le fait. Devant son agent, le libéré se cherche activement un emploi, mais la tâche est fort complexe, étant donné son passé criminel (quelques-uns avoueront ouvertement profiter des prestations du B.E.S. et vouloir continuer), il a de bonnes relations avec son milieu familial et il n'enfreint jamais la loi.

Comment pourrait-il en être autrement quand on sait que le libéré peut voir sa libération suspendue du seul fait qu'il enfreint une loi ou une condition de sa libération conditionnelle comme de sa surveillance obligatoire?

Dans ce jeu de méfiance du libéré envers son agent et de contrôle de ce dernier envers les libérés qu'on lui confie, il n'existe plus, dans la majorité des cas, de conversation où le libéré et l'agent entrent en contact simple, sans mise en garde, sur des sujets qui impliquent les valeurs profondes du libéré. Il est certain qu'on pourra croire l'inverse dans plusieurs cas, mais il est trop évident que l'ex-détenu accepte cette conversation davantage pour ne pas être en mauvais termes avec son agent que parce qu'il y croit vraiment. Comme le mentionne McCleary, l'agent veut faire de la thérapie par souci de professionnalisme alors qu'il s'aperçoit qu'il ne peut le faire qu'avec des ex-détenus spéciaux, dans un contexte spécial ${ }^{5}$. Dans ces cas spécifiques, l'agent se référera très souvent

5. McCleary, Richard (1978), Dangerous Men, the Society of Parole, London, Sage Publications, p. 106. 
à une agence spécialisée parce qu'il se sent mal préparé à affronter ces cas particuliers (schizophrène, paranoïaque, etc.).

L'agent, qui est perçu d'abord par son rôle de contrôle avant d'être un aide potentiel, en cas de besoin, se heurtera donc à un ex-détenu distant qui n'acceptera jamais d'étaler ses problèmes d'alcoolisme, de drogue ou d'ordre sexuel, si ce n'est en termes génériques, par peur d'être surveillé de plus près, de voir ses gestes découverts et d'avoir à subir une sanction quelconque. L'ambiguïté de notre rôle, que le libéré saisit très bien, habitué qu'il est à un contrôle permanent de tous ses gestes, détruit toute possibilité de relation thérapeutique avant même qu'elle ne débute.

L'ex-détenu qui acceptera d'entrer dans une certaine relation d'aide visant à transformer graduellement certaines de ses valeurs est rarissime. C'est également celui qui a le moins de crainte de voir ses difficultés d'adaptation impliquer un contrôle plus rigoureux et par conséquent celui qui côtoie le moins la délinquance si pourchassée par l'agent.

Il faut donc accepter que le double rôle de l'agent de libération conditionnelle connaisse certains échecs évidents et que le travail de thérapeute qu'il tente d'exercer en toute bonne foi va choir dans un terrain peu propice. Il est toujours flatteur de se rabattre sur cette fonction prestigieuse dans notre société, mais le climat de méfiance dont nous avons parlé, le manque de préparation que ressentent à tort ou à raison, nombre d'agents, et la nonréceptivité quasi générale des libérés, nous portent à conclure qu'aucune véritable relation thérapeutique ne peut s'amorcer entre l'ex-détenu et son agent de libération conditionnelle.

\section{Une relation d'aide est-elle possible?}

Notre travail quotidien s'effectue donc à un niveau beaucoup plus empirique où, d'une part, plus de la moitié de notre temps est occupée à transmettre de l'information par écrit à la Commission des libérations conditionnelles, etc. D'autre part, dans notre relation avec le sujet on s'efforce de vérifier avant tout, si la recherche d'emploi qu'il effectue est adéquate, s'il à l'intention de quitter la région ou s'il doit changer de domicile. Nous le suivons donc dans le déroulement des événements concrets et importants de sa vie bien plus qu'au niveau des valeurs que l'ex-détenu véhicule à ces occasions, et cela à cause de la distance entre le libéré et l'agent qui subsiste malgré toute la bonne volonté de ce dernier.

Quoiqu'il soit difficile de quantifier l'impact de l'agent sur 
les démarches entreprises par le libéré, il est fort possible que les pressions exercées constamment par le premier en ce qui a trait à la recherche d'emploi, ou la stabilité d'une relation familiale, incitent le second à présenter ce panorama et à faire certains efforts véritables en ce sens. Cependant, c'est davantage la crainte des représailles qu'un changement authentique d'attitude qui sera à la base du respect des conditions de la libération. Ainsi, nombre de libérés mèneront deux systèmes de valeurs parallèles qu'ils présenteront selon que leur interlocuteur sera l'agent de libération conditionnelle ou l'ancien complice avec lequel ils ont commis l'infraction qui les a conduits au pénitencier.

Il nous semble donc évident que l'aide que peut apporter l'agent de libération conditionnelle ne se situe pas à l'échelle d'une relation thérapeutique, mais plutôt à un niveau très empirique. En effet, sans se confier vraiment à son agent, l'ex-détenu le plus démuni se rendra souvent chez celui-ci afin qu'il l'accompagne, l'introduise ou le supporte dans ses démarches auprès des autres organismes gouvernementaux, ou para-gouvernementaux (Bienêtre social, Centre de main-d'œuvre, Centre d'accueil, etc.).

Comme l'écrit Inkeri Anttila à Ia fin de son article :

Some offenders would not need any help [. . .]. In other cases, maybe help is needed in searching for a job and a place for living, or in order to provide money to pay a few week's rent in advance. In very rare cases, some counselling and/or psychiatric treatment would be adbisable ${ }^{6}$.

Un contrôle efficace?

L'objectif premier de notre travail demeure donc, jusqu'à présent, le contrôle de l'activité de l'ex-détenu et la protection de la société menacée en certaines occasions par les délits commis contre la propriété et contre les personnes?

L'agent de libération conditionnelle rencontre le libéré à toutes les deux semaines à son bureau ou chez ce dernier afin de faire le point sur les activités passées du sujet et sur les projets que l'un ou l'autre croit important de mettre en marche. Ainsi, particulièrement au cours des premières semaines, on parlera généralement des démarches que le libéré aura faites pour se

6. Anttila, Inkeri (1975). «Probation and Parole : Social Control or Social Service? », in International Journal of Criminology and Penology, vol. 3, p. 84 .

7. Un débat sur l'équité de la distribution des biens dans une société comme la nôtre pourrait être entendu en d'autres lieux, mais nous refusons de débattre ce sujet à l'intérieur de la présente réflexion. 
trouver un emploi et des moyens qu'il devrait utiliser afin d'être embauché, des ressources financières qu'il doit se trouver à court terme, et peut-être de l'endroit où il aimerait aller résider considérant sa situation économique, sociale et familiale.

Nous savons, par ailleurs, que l'individu qui quitte le pénitencier est très souvent sollicité par ses anciens complices pour renouer avec l'activité criminelle quand ce n'est pas lui-même qui prépare un nouveau délit. Rappelons qu'au moins un détenu sur deux purge plus d'une sentence suite à un délit, ce qui ne comprend pas ceux qui commettent des infractions criminelles sans être reconnus coupables.

Pourtant, de façon générale il ne faut pas croire que l'exdétenu élaborera longuement sur les liaisons qu'il a eues ou qu'il maintient avec ses anciens complices. Même les membres de la famille que nous rencontrons régulièrement lors de nos visites à domicile tairont ce genre d'information s'ils la connaissent. En effet, même si la mère ou l'épouse d'un libéré n'encourage pas ce dernier à renouer avec le milieu criminel, elles hésiteront longuement avant d'informer l'agent de libération conditionnelle des illégalités commises. Elles ne veulent pas voir leur fils, ou leur mari, retourner au pénitencier et, souvent, elles espèrent aussi que leurs propres avertissements suffiront à le ramener sur le chemin de la légalité.

Devant cet état de faits, l'agent a-t-il vraiment un choix, malgré certains soupçons, de croire ou de ne pas croire le libéré qui accepte de répondre à ses questions? Est-ce qu'un seul soupçon devrait être suffisant pour suspendre la libération conditionnelle ou la surveillance obligatoire d'un individu? Une fois faites quelques vérifications de routine, nous devons laisser l'ex-détenu tenter de réintégrer la société, et ce, même si nous ne croyons pas qu'il fasse de véritables tentatives en ce sens, même si nous soupçonnons qu'il prépare un délit et même s'il a six, sept ou dix vols par effraction à son actif. Si nous arrêtions tous les ex-détenus soupçonnés de participer à une action illégale, bien peu d'entre eux demeureraient en liberté, étant donné leur style de vie et surtout leurs activités criminelles antérieures. Bref, le libéré a tout le temps désiré pour commettre un délit entre deux visites de son agent et ce dernier a le choix de croire ou de douter de la personne qu'il rencontre mais ne peut agir véritablement sans preuve plus ou moins tangible. 
De plus, peut-on attendre d'un individu ayant une formation en relations humaines qu'il joue un jeu plus ou moins assimilable à celui d'un détective? Son rôle devient de plus en plus administratif et ses compétences sont rarement mises à l'épreuve. L'incarcération est perçue comme la solution par excellence en vue de minimiser les risques causés par la mise en liberté des détenus.

\section{Une liberté croissante}

Quand le comportement de l'ex-détenu nous apparaît conforme aux normes qu'il doit suivre et qu'aucun problème particulier ne se présente, l'agent diminue graduellement la fréquence de ses rencontres, passant d'abord à une visite tous les trois semaines puis tous les mois. Le libéré semble ressentir une pression moins grande et une confiance accrue de la part de son agent, mais la récidive demeure possible en tout temps. Autant avons-nous connu des récidivistes qui étaient en liberté conditionnelle depuis plus de deux ans, quoique moins nombreux, autant en avons-nous connus qui ont récidivé après avoir passé quelques semaines dans la communauté. Un agent quelque peu expérimenté pourra faire un pronostic sur la durée du passage de l'ex-détenu en communauté et ne pas faire trop d'erreurs, mais ce n'est pas le rythme des rencontres avec le libéré qui aura une influence notable sur la récidive. Nous ne croyons donc pas que la présence d'un agent qui veut aider et surveiller un ex-détenu modifiera sensiblement le comportement de ce dernier.

Déjà au deuxième congrès de criminologie du Québec en 1968 on rapportait une étude effectuée à San Francisco où l'on avait évalué la récidive de quatre groupes d'ex-détenus. Le premier groupe était vu toutes les semaines par des agents qui possédaient une maîtrise en consultation psychiatrique, le second groupe était vu au même rythme par un groupe d'agents volontaires représentatifs de l'ensemble, les troisième et quatrième groupes étaient vus une fois par mois, respectivement par les agents possédant une maîtrise en consultation psychiatrique et par les agents volontaires. L'auteur conclut :

After several years, no statistically significant difference was to be found in the recidivism rates between the four groups in terms of new offenses ${ }^{8}$.

8. La société de criminologie du Québec (1969). Deuxième Congrès de criminologie du Québec, Montréal, Librairie Beauchemin, p. 20. 


\section{C) LA SENTENCE DÉTERMINÉE}

Les initiateurs du courant thérapeutique et ceux qui ont défendu avec ferveur le rôle de l'agent de libération conditionnelle tel qu'on le connait aujourd'hui avaient probablement prévu des résultats plus probants que ceux que l'on a décrits jusqu’à présent. À l'heure actuelle, chacun se pose des questions sur cette fonction et plusieurs cercles d'intellectuels chevronnés ne veulent même plus se pencher sur les problèmes quotidiens que nous avons énoncés, prétextant l'inopportunité de ce travail. Pourtant, près des exdétenus, mêlés à leurs problèmes journaliers et vivant souvent en même temps qu'eux leurs angoisses et leurs aspirations, il nous semble que certains services devraient leur être rendus parce qu'ils en ressentent un besoin urgent et que la population a ce devoir envers eux après les avoir exclus momentanément de la vie sociale.

\section{Répondre à un besoin spécifique}

Lorsqu'un détenu quitte le pénitencier, démuni, sans argent et sans famille prête à l'accueillir, l'agent demeure son point de contact privilégié à l'extérieur du milieu criminel, et ce, malgré I'hostilité que peuvent dégager les premières rencontres. Très souvent, sans connaissance des mécanismes bureaucratiques auxquels il se frappe constamment, et ignorant les services que peut lui offrir la communauté, le libéré se référera à son agent afin de pouvoir acquérir les ressources nécessaires (logement, argent, emploi, paiement d'anciennes contraventions, etc.) pour survivre dans un monde d'où il est trop souvent exclu.

Toutefois, le libéré apprendra rapidement à négocier avec tous ces services et l'agent, qui fut au départ une ressource privilégiée sans préjugé trop négatif vis-à-vis de l'ex-détenu, verra son impact diminuer et souvent se perdre parmi tous les agents sociaux (responsable du B.E.S., travailleurs sociaux, etc.) qui côtoient ce même individu.

Ayant déjà démontré antérieurement l'inefficacité de la thérapie et du contrôle offert par l'agent de libération conditionnelle auprès de l'ex-détenu et, d'autre part, croyant que ces derniers ont certains besoins légitimes que la population doit offrir, nous favorisons un déplacement de l'objectif du travail de l'agent. En effet, au lieu de forcer une relation dont le libéré ne veut pas, il serait préférable de tenter de répondre aux besoins de celui qui 
entre démuni dans une société qu'il a quittée pendant un certain temps.

Nous croyons également qu'un tel changement d'objectif nécessite l'adoption de la sentence déterminée afin que toute relation cœrcitive entre le libéré et son agent soit abolie. Évidemment, la sentence déterminée comporte des avantages comme des inconvénients. Ainsi, la Commission des libérations conditionnelles n'existant plus, elle ne pourrait plus corriger une fausse perception du juge qui aurait condamné un inculpé à une sentence plutôt sévère comme cela est possible actuellement.

Par contre, la sentence déterminée limiterait l'arbitraire de la sanction prononcée par le juge à une marge minimale. Mais, beaucoup plus important, la relation entre le détenu et son agent serait sensiblement modifiée à l'intérieur comme à l'extérieur des institutions pénitentiaires. Le détenu ne craignant plus la portée corcitive de sa relation avec son agent, puisque ce dernier n'aurait plus de pouvoir à ce niveau, échangerait avec plus de franchise et cesserait de jouer le rôle lui permettant soit de quitter le pénitencier avant sa surveillance obligatoire, soit de ne pas réintégrer l'institution suite à une mauvaise conduite.

Il est fort possible que le retrait de la libération conditionnelle dans sa forme actuelle ait un effet sur la tension qui existe dans les institutions. Par contre, certains programmes de travail mieux rétribués, des contacts familiaux plus fréquents ou des changements d'institution plus accessibles pourraient concourir à éliminer cette tension.

\section{La relation agent/ex-détenu}

Le service que nous proposons d'offrir à l'ex-détenu ne s'adresserait pas nécessairement à tous ceux qui quittent le pénitencier, mais uniquement à ceux qui désirent profiter d'une aide disponible spécialement offerte à l'ex-détenu. Ainsi, puisque celuici n'aurait plus à craindre son agent, il serait davantage prêt à élaborer sur ses besoins et l'agent serait plus en mesure de lui rendre les services adéquats. Cependant, l'objectif de ces rencontres ne serait plus d'établir une relation «thérapeutique », sans qu'une demande provienne du libéré, mais uniquement de travailler de concert avec ce dernier afin de l'aider à répondre à des besoins spécifiques (logement, travail, argent, rencontre de créanciers, etc.) lui permettant, s'il le veut, de réintégrer graduellement Ia société. 
En effet, ce genre de services qu'utiliserait le libéré, selon ses besoins, éliminerait ceux qui n'ont pas encore décidé de ne plus commettre de délit et ceux qui ont un tissu social et familial prêt à les accueillir, indépendamment de la présence d'un agent de libération conditionnelle.

Les derniers peuvent réintégrer seuls la société et la tâche actuelle de l'agent se résume à des aspects purement techniques et bureaucratiques. Quant aux premiers, ils se tourneront davantage vers leurs anciens complices et commettront fort probablement de nouveaux délits, mais ce n'est pas notre présence actuelle qui les en empêchera. L'illusion d'une protection créée par la présence de l'agent disparaîtrait mais la réalité ne serait guère modifiée.

Bref, pour la première fois, l'ex-détenu se présenterait à son agent sur une base volontaire et les deux interlocuteurs travailleraient conjointement en vue d'un objectif commun. Ainsi, au lieu de rencontrer son agent dans un climat de méfiance et de lui cacher nombre de ses activités, le libéré rencontrerait son agent dans un contexte plus sain où, ensemble, ces deux partenaires viseraient la solution d'un problème spécifique. Enfin, au lieu de travailler l'un contre l'autre, ils travailleraient l'un avec l'autre.

\section{Réactions sociales}

L'adoption de la sentence déterminée et du nouveau rôle de l'agent de libération conditionnelle exigerait quelques modifications notamment vis-à-vis des sentences prononcées par les juges. Il est fréquent d'entendre au sein du système judiciaire que les sentences ont été prolongées parce qu'on a anticipé l'octroi d'une libération conditionnelle. Un certain réajustement serait donc nécessaire à ce chapitre dans l'optique le plus classique de la sentence déterminée.

D'autre part, la population, qui connaît peu dans l'ensemble le système judiciaire et de façon plus spécifique le rôle de l'agent de libération conditionnelle, acceptera sans trop de heurts la vision que nous avons tenté de présenter. De plus, comme nous le mentionnons précédemment, ce n'est pas la présence d'un agent telle qu'on la conçoit actuellement qui limite de façon notable le nombre de récidivistes.

Par contre, le coût financier d'une telle opération serait probablement moindre que le coût actuel d'un service de libérations conditionnelles et, surtout, le service rendu à l'ex-détenu qui 
se prévaudrait de son droit serait largement supérieur à celui qu'on lui offre actuellement et auquel il n'est généralement pas intéressé.

Nous croyons qu'un tel changement d'orientation permettrait d'éliminer une importante part de l'aspect bureaucratique de notre travail alors que le service désiré par notre clientèle serait amélioré. CONCLUSION

La surveillance des agents du comportement social des exdétenus a souvent droit de regard sur plusieurs activités, acceptables au niveau des normes sociales mais que le surveillant doit faire cesser (consommation légère de drogue, fréquentation d'individus non désirables, consommation d'alcool, activités de loisirs dispendieuses, etc.). Ainsi, un libéré qui réussira à traverser sa période en libération conditionnelle ou en survellance obligatoire sans difficulté le devra souvent, soit à sa capacité de cacher tous ses agirs délinquants ou soit à un comportement que l'on pourrait qualifier d'irréprochable en termes de respect des lois sociales. Nous demandons à l'individu libéré du pénitencier d'avoir des pratiques plus conformes aux normes sociales que celles d'un grand nombre d'individus sans casier judiciaire.

Lorsque le libéré est surpris en infraction, la peine imposée sera souvent plus sévère que celle qui aurait été imposée à un autre individu ne possédant pas de casier judiciaire, et ce, même si cette nouvelle infraction est tout à fait différente des infractions précédentes.

À la question : "Quelle est la difficulté majeure rencontrée au cours de la période de surveillance effectuée par votre agent? », nombre de libérés terminant leur sentence répondront que c'est avant tout la crainte constante de retourner au pénitencier. Les rencontres régulières avec l'agent de libération conditionnelle sont davantage acceptées et ne posent pas ce problème quotidien.

D'autre part, la Commission des libérations conditionnelles, qui devrait avant tout permettre et aider l'ex-détenu à réintégrer la société, semble s'être éloignée de plus en plus de cette mission première et avoir davantage adopté un rôle répressif. Bora Laskin, juge en chef de la Cour suprême du Canada, citait le juge Rand dans un de ses jugements en ces termes :

La seule réfutation proposée à ce sujet consiste à dire que la Commission, étant un "organe administratif », peut en fait agir comme elle l'entend. Toutefois, en ce domaine, nous sommes trop prisonniers des mots. D'un point de vue administratif, dans la promulgation de législation par pouvoir 
délégué, le principe a une application limitée; toutefois, dans la complexité de l'activité gouvernementale d'aujourd'hui, une soi-disant commission administrative peut être chargée non seulement de fonctions d'administration et d'exécution, mais aussi de fonctions judiciaires et c'est sur ces fonctions que nous devons porter notre attention. Lorsqu'elles sont d'une nature judiciaire, elles touchent à l'extinction ou à la modification de droits ou d'intérêts privés. Le plein exercice des droits en question, dont certains sont reconnus et d'autres conférés par la loi, dépend des conclusions de la Commission; tostefois, ces droits ne sont pas créés par la Commission, et leur jouissance n'est pas davantage soumise à la volonté pure et «simple de la Commission »; l'Association ne peut être privée de leur jouissance qu'au moyen d'une procédure inhérente à l'action judiciaire?

Celui-ci poursuivait relativement à la véritable identité de la Commission des libérations conditionnelles :

Le fait brutal est que la Commission revendique un pouvoir tyrannique qui est, à mon avis, sans précédent parmi les organismes administratifs habilités à statuer sur la liberté individuelle. Elle réclame un pouvoir absolu sur la personne d'un détenu, presque comme s'il s'agissait d'une simple marionnette. Selon ses prétentions, la Commission peut appliquer les normes législatives suivant son jugement et sans avoir aucun compte à rendre aux tribunaux. Il faut la croire sur parole quand elle déclare agir équitablement, car elle n'est pas tenue de donner la moindre indication du motif de suspension ou de révocation de la libération conditionnelle. On prétend que tout ceci ressort de la «Loi sur la libération conditionnelle de détenus » et, de plus, que c'est de cette façon que cette Cour a envisagé les pouvoirs de la Commission dans J'arrêt Howarth ${ }^{10}$.

Quoique certaines modifications ont été appliquées au cours des dernières années, il n'en demeure pas moins que la Commission des libérations conditionnelles s'impose davantage en tant qu'organisme répressif plutôt qu'un instrument d'aide à l'ex-détenu. Trop souvent, cette Commission cherche les difficultés que poserait la réinsertion sociale du détenu au lieu de chercher les avantages et les valeurs de celui-ci, sur lesquels une réintégration sociale pourrait se construire. Nous devons donc nous questionner sur l'aide qu'apporte la Commission des libérations conditionnelles au détenu qui désire retourner dans la société.

9. Laskin, Bora (1975). Rapport de la Cour suprême 2e vol. (Mitchell contre la Reine), p. 580 (tiré de l'arrêt l'Alliance des professeurs catholiques de Montréal contre la Commission des Relations ouvrières du Québec, 1953). 10. Laskin, Bora (1975). Op. cit. (Mitchell contre la Reine), p. 577. 
Au cours de cet article, nous avons longuement démontré que le travail quotidien auprès des ex-détenus nous incite chaque jour davantage à nous interroger sur la valeur d'une « relation thérapeutique » que l'on voudrait imposer dans la majorité des cas à ceux-ci. Il nous a semblé également qu'une rencontre à toutes les deux semaines n'inquiétait nullement le libéré qui désirait récidiver et qu'au contraire ceci le sécurisait à l'occasion devant les interrogations policières. Ainsi, les deux objectifs fondamentaux de notre travail n'auraient connu qu'un léger succès auprès des libérés, qui, de toute façon, n'auraient probablement jamais récidivé.

Nous proposons donc de modifier l'objectif de notre travail en créant un service d'aide aux détenus que ceux-ci utiliseraient sur une base volontaire et selon leurs besoins. Ayant retirè à l'agent de libération conditionnelle son pouvoir cœrcitif, celui-ci deviendrait un membre de la communauté privilégié et spécialement dévoué à la cause de l'ex-détenu qui cherche à répondre à des besoins spécifiques, qui sont légitimes quand on aspire à réintégrer adéquatement la société après une incarcération de deux ans ou plus.

Actuellement, l'agent donne souvent l'impression d'apporter une aide thérapeutique au libéré et de protéger la société alors que nous croyons que l'efficacité de son travail se résume essentiellement aux efforts qu'il fait conjointement avec l'ex-détenu relativement à la recherche d'un logement, d'un travail, etc.

Le changement d'orientation que nous proposons ne réglera pas le problème des récidivistes mais il dévoilera la véritable efficacité de la fonction d'agent et proposera des instruments au libéré qui veut définitivement quitter le milieu criminel.

Il n'en demeure pas moins que l'individu qui réussit sa libération conditionnelle, le devra avant tout à lui-même et non aux divers intervenants qui le côtoient.

Pour le moment la question qui se pose a trait à la justification même de l'existence du système de libération conditionnelle. 